\title{
Research on A Novel MPP Tracking Strategy Reaction to Rapid Change of Light Intensity
}

Chunhu Sun ( $\nabla$ sunchunhu@126.com )

chaohu university

Jing Ling

chaohu university

Tingting Liu

chaohu university

\section{Research Article}

Keywords: MPPT,Power prediction method,Optimal gradient method, Misjudgment phenomenon, Oscillation phenomenon

Posted Date: September 21st, 2021

DOI: https://doi.org/10.21203/rs.3.rs-907438/v1

License: (c) (1) This work is licensed under a Creative Commons Attribution 4.0 International License. Read Full License 


\title{
Research on A novel MPP tracking strategy reaction to Rapid change of light intensity
}

\author{
Sun Chunhu*, Ling Jing, Liu Tingting \\ (School of Electronic engineering , Chaohu University, Chaohu 238000 ,China)
}

\begin{abstract}
For the misjudgment and oscillation in the traditional MPP tracking process when the light intensity changes rapidly, a novel improved optimal gradient method for power prediction is proposed, based on the research of the traditional power prediction method and the optimal gradient method.In this article, the working principle, advantages and disadvantages of the traditional power prediction method and optimal gradient method are firstly analyzed; then a novel power prediction method with high-precision power calculation, as we as an improved optimal gradient method are proposed. Finally, Simulink simulation analysis is carried out on the proposed MPP tracking strategy when the light intensity changes rapidly. The simulation results show that when the light intensity changes rapidly, the novel power prediction improvement is adopted. The optimal gradient method can not only eliminate the misjudgment phenomenon and oscillation phenomenon, but also the power calculation is more accurate. The introduction of the variable step factor can make the system output more power, which can well realize the fast tracking of MPP.
\end{abstract}

Keywords: MPPT;Power prediction method;Optimal gradient method; Misjudgment phenomenon; Oscillation phenomenon

\section{Introduction}

In order to increase the output power of photovoltaic power generation systems, many scholars have conducted in-depth research on the maximum power point tracking (MPPT) algorithm in recent years. From the original MPPT algorithm, such as constant voltage method, disturbance observation method, and conductance increment method, to the later improved MPPT algorithm, such as variable-step MPPT control method, fuzzy logic control method, neural network control method, etc. Now, the improved MPPT algorithm has made new progress. Literature [1] reviews the research of MPPT algorithm ,the advantages and disadvantages of the common photovoltaic power generation MPPT algorithm under uniform light conditions are discussed and compared, and the development trend of the MPPT algorithm is pointed out. Literature [2] proposed the interleaved parallel Boost circuit as the front-end circuit, which shortened the tracking time and improved the system efficiency. The improved variable-step conductance increment method has faster tracking speed and better stability. Literature [3] proposed a self-optimizing perturbation observation method based on hysteresis comparison, which can take into account the tracking speed and tracking accuracy of the system, also effectively reducing system oscillations and avoiding misjudgments. Literature [4] improved the traditional power prediction disturbance observation method, and proposed a power prediction method based on Lagrangian linear interpolation, which greatly reduces the system error, can adjust the control strategy in time, and effectively avoid the occurrence of misjudgment.

Although these traditional and improved MPPT algorithms can achieve maximum power point (MPP) tracking well, they will temporarily fail under the condition of rapid changes of light intensity, which usually accompanied by oscillation and misjudgment phenomenon. Therefore, it 
is impossible to track MPP accurately. For this reason, many scholars have also conducted research and exploration on this situation. The literature [5-6] established a variable weather parameter (VWP) interval centered on the control amount corresponding to the ideal MPP, and limited the optimization range of the MPP within this interval, which improved the rapidity of system tracking and decreased The optimal step length ensures the accuracy of system tracking. Literature [7-9] Based on the basic circuit equations of solar cells, a mathematical model between the disturbance step length of the MPPT algorithm and the light irradiation intensity, ambient temperature, maximum power point and sampling period is constructed. This disturbance step mathematical model can be effective to avoid the occurrence of misjudgment, it shows high reliability and practicability. Literature [10-11] studied the mathematical model of photovoltaic array characteristics and the reasons for the instability of the conventional maximum power tracking method when the light intensity changes rapidly, and proposed to insert a measurement at two sampling points to suppress the influence of light intensity changes on the photovoltaic system. The maximum power tracking method effectively avoids misjudgments and improves the stability of the system. Literature [12-13] improved the traditional dP-P\&O algorithm, and proposed the dP-P\&O-dP maximum power tracking algorithm, which effectively avoided the occurrence of misjudgment, and the variable-step tracking method took account of the rapidity and stability of tracking well. Literature [14-16] proposed an improved INC algorithm. When the light intensity rises rapidly, the variable step length INC algorithm is used to track the maximum power point. When the light intensity drops rapidly, the load curve and the IV characteristic curve are used to quickly track the maximum power point. The algorithm shows fast response speed and high tracking accuracy under the rapid change of light intensity. Literature [17-20] proposed the use of an improved power prediction method to adapt to rapid changes in light intensity. Although it can reduce misjudgments, the modeling process is not accurate enough and accompanied with some errors. For example, when the light intensity changes non-uniformly, it still cannot avoid misjudgments and oscillations. Literature [21] proposed a variable-step perturbation observation method that simplified the gradient method. Although the tracking accuracy is improved, the step size factor is fixed and the design is more complicated. Literature [22] proposed a three-stage variable step size perturbation observation method, which improved the tracking speed, but the third stage still has oscillation due to the fixed step length.

Based on the above literature, in order to adapt to the tracking requirements of the MPPT algorithm under rapid changes in light intensity, this article proposes a new improved optimal gradient method for power prediction, which can be effective when the light intensity changes uniformly and non-uniformly, as well as avoiding misjudgment and oscillation, and improve the tracking speed and accuracy.

\section{Traditional power prediction method}

The traditional power prediction method and the improved power prediction method are studied, and the working principle and the existing advantages and disadvantages of the traditional power prediction method and the improved power prediction method are respectively analyzed.

\subsection{Traditional power prediction method}

The traditional power prediction method is shown in Figure 1, and the working principle is as follows. 


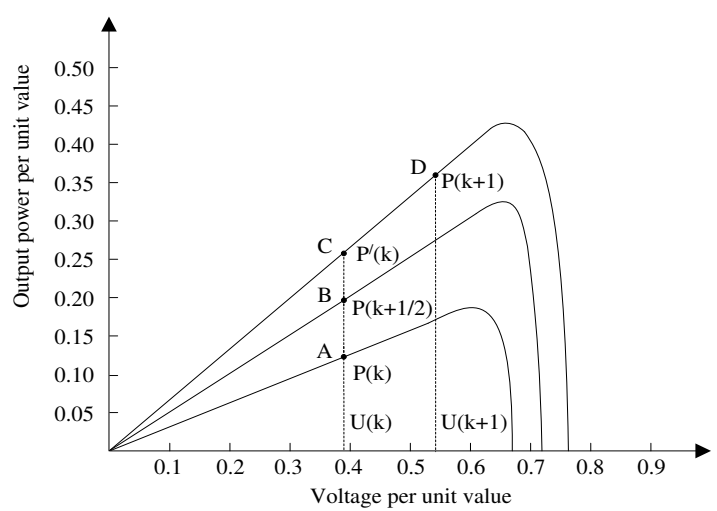

Figure 1 The traditional power prediction method

Assuming that in the current system disturbance voltage is $U(k)$, the output power is $P(k)$, and the operating point is at point $A$ in the figure; the disturbance voltage is kept unchanged at $U(k)$, and the output power is sampled after half a sampling period. When the operating point is at point $\mathrm{B}$, the output power is $\mathrm{P}(\mathrm{k}+1 / 2)$; if the illumination changes uniformly, the output power will also change uniformly. It can be predicted that when the disturbance voltage is $U(k)$ unchanged, After 1 sampling period, the output power $\mathrm{P}^{\prime}(\mathrm{k})$ at operating point $C$ can be expressed as:

$$
P^{\prime}(k)=2 P(k+1 / 2)-P(k)
$$

After 1 sampling period, when the disturbance voltage becomes $U(k+1)$, the operating point is at point $D$, and the measured output power is $P(k+1)$; if the output power $P^{\prime}(k)<P(k+1)$, then increase the disturbance voltage; if the output power $\mathrm{P}^{\prime}(\mathrm{k})>\mathrm{P}(\mathrm{k}+1)$ at this time, then reduce the disturbance voltage; because point $\mathrm{C}$ and point $\mathrm{D}$ are on the $\mathrm{PU}$ characteristic curve under the same light conditions Therefore, the use of power prediction method will not cause misjudgment.

Although the traditional power prediction method can effectively avoid the misjudgment phenomenon, it is based on the uniform change of illumination. Therefore, when the illumination changes non-uniformly, the traditional power prediction method will be invalid and the misjudgment phenomenon cannot be avoided.

\subsection{Improved power prediction method}

In view of the non-uniform changes in illumination, the traditional power prediction method cannot solve the problem of misjudgment. Some scholars have proposed an improved power prediction method, as shown in Figure 2.

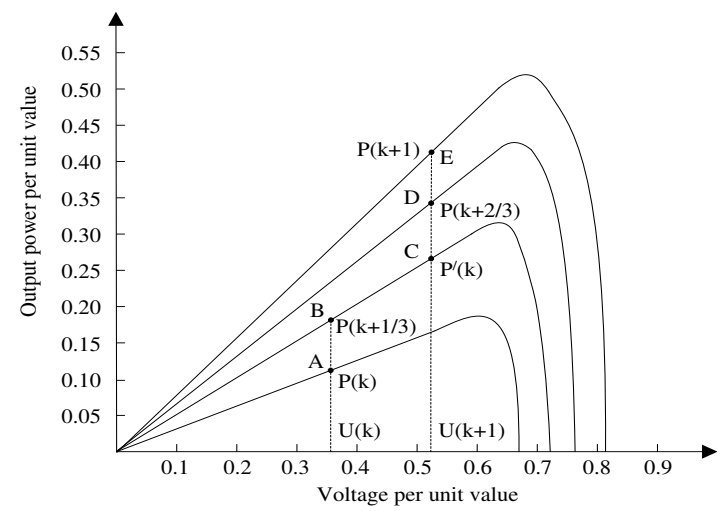

Figure 2 Improved power prediction method 
Assuming that the current system disturbance voltage is $U(k)$, the output power is $P(k)$, and the operating point is at point $A$ in the figure; the disturbance voltage is maintained at $U(k)$ unchanged, and the output power is sampled after $1 / 3$ sampling period, The operating point is at point $B$, and the output power is $P(k+1 / 3)$; after another $1 / 3$ sampling period, the disturbance voltage is changed to $U(k+1)$, and the operating point is at point $D$, The output power is $P(k+2 \beta)$; keep the disturbance voltage $U(k+1)$ unchanged, and then sample the output power after $1 / 3$ sampling period. At this time, the operating point is at point $E$, and the output power is $P(k+1)$; assuming that the power at point $C$ is $P^{\prime}(k)$, the change in output power from point $A$ to point $B$ is defined as $a_{1}$, then:

$$
\mathrm{a}_{1}=P(\mathrm{k}+1 / 3)-P(k)
$$

In the same way, the output power changes $\mathrm{a}_{2}$ and $\mathrm{a}_{3}$ from point $\mathrm{C}$ to point $\mathrm{D}$ and from point $D$ to point $E$ can be defined.

$$
\begin{aligned}
& \mathrm{a}_{2}=P(\mathrm{k}+2 / 3)-P^{\prime}(k) \\
& \mathrm{a}_{3}=P(\mathrm{k}+1)-P(k+2 / 3)
\end{aligned}
$$

The change in output power from point $A$ to point $B$, the change in output power from point $C$ to point $D$, and the change in output power from point $D$ to point $E$ are all caused by continuous changes in illumination, so the following relationship can be obtained:

$$
2 \mathrm{a}_{2}=\mathrm{a}_{1}+\mathrm{a}_{3}
$$

Formula (2) (4) are put into formula (5) to get:

$$
2 P(\mathrm{k}+2 / 3)-2 P^{\prime}(k)=P(\mathrm{k}+1 / 3)-P(\mathrm{k})+P(\mathrm{k}+1)-P(k+2 / 3)
$$

According to formula (6), the output power at point $\mathrm{C}$ can be solved as:

$$
P^{\prime}(k)=\frac{3 P(\mathrm{k}+2 / 3)-P(\mathrm{k}+1 / 3)+P(\mathrm{k})-P(k+1)}{2}
$$

And point $B$ and point $C$ are the operating points on the same $P-U$ characteristic curve. Therefore, it can be concluded that:

If $P(k+1 / 3)>P /(k)$, then reduce the disturbance voltage; if $P(k+1 / 3)<P^{\prime}(k)$, then increase the disturbance voltage; it can be seen that the improved power prediction method is adopted. It solves the misjudgment phenomenon when the illumination changes unevenly, and the adaptability is stronger.

However, the improved power prediction method also has certain error defects. This error is mainly due to the fact that the operating points $A$ to $D$ do not work under the same disturbance voltage, which causes the accuracy of the power calculation to decrease.

\section{Novel power prediction method}

In order to improve the accuracy of power calculation of the improved power prediction method and reduce the error, a novel power prediction method is proposed in the article on the basis of the improved power prediction method, as shown in Figure 3. 


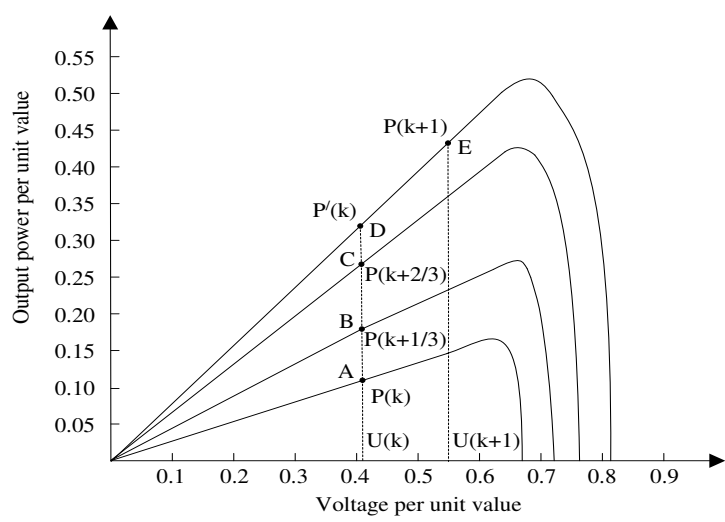

Figure 3 Novel power prediction method

Assuming that the current system disturbance voltage is $\mathrm{U}(\mathrm{k})$, the output power is $\mathrm{P}(\mathrm{k})$, and the operating point is at point $A$ in the figure; the disturbance voltage is maintained at $U(k)$ unchanged, and the output power is sampled after $1 / 3$ sampling period, The working point is at point $B$ at this time, and the output power is $P(k+1 / 3)$; continue to keep the disturbance voltage as $U(k)$ unchanged, and after $1 / 3$ of the sampling period, the working point is at point $C$, The output power is $\mathrm{P}(\mathrm{k}+2 / \beta)$; the output power is sampled after $1 / 3$ sampling period, the disturbance voltage becomes $U(k+1)$, the working point is at point $E$, and the output power is $P(k+1)$; Assuming that the power at point $\mathrm{D}$ is $\mathrm{P}^{\prime}(\mathrm{k})$, and the change in output power from point $A$ to point $B$ is defined as $b_{1}$, then:

$$
\mathrm{b}_{1}=P(\mathrm{k}+1 / 3)-P(k)
$$

In the same way, the output power changes $b_{2}$ and $b_{3}$ from point $B$ to point $C$ and from point $C$ to point $D$ can be defined.

$$
\begin{gathered}
\mathrm{b}_{2}=P(\mathrm{k}+2 / 3)-P(\mathrm{k}+1 / 3) \\
\mathrm{b}_{3}=P^{\prime}(\mathrm{k})-P(k+2 / 3)
\end{gathered}
$$

The change in output power from point $A$ to point $B$, the change in output power from point $B$ to point $C$, and the change in output power from point $C$ to point $D$ are all caused by continuous changes in illumination. At the same time, points $A$ to $D$ are all working under the same disturbance voltage $U(k)$, the accuracy of power calculation is improved. In the same way, the following relationship can be obtained:

$$
2 b_{2}=b_{1}+b_{3}
$$

Formula (8) $\sim(10)$ are put into formula (11) to get:

$$
2 P(\mathrm{k}+2 / 3)-2 P(k+1 / 3)=P(\mathrm{k}+1 / 3)-P(\mathrm{k})+P^{\prime}(\mathrm{k})-P(k+2 / 3)
$$

According to formula (12), the output power at point $\mathrm{C}$ can be solved as:

$$
P^{\prime}(k)=3 P(\mathrm{k}+2 / 3)-3 P(\mathrm{k}+1 / 3)+P(\mathrm{k})
$$

And point $D$ and point $E$ are the operating points on the same $P-U$ characteristic curve. Similarly, it can be concluded that:

If $P(k+1)>P^{\prime}(k)$, then increase the disturbance voltage; if $P(k+1)<P /(k)$, then reduce the 
disturbance voltage; it can be seen that the novel power prediction method not only has improved the advantages of the power prediction method, at the same time, the operating points $A$ to $D$ are all working under the same disturbance voltage, which effectively improves the accuracy of power calculation.

\section{Optimal Gradient Method}

Although the power prediction method has solved the problem of misjudgment, the problem of oscillation has not been effectively solved. The oscillation problem is caused by the unreasonable disturbance step size control, so variable step size control needs to be adopted, and the optimal gradient method is a widely used variable step size control method. Based on the analysis of the working principle and advantages and disadvantages of the optimal gradient method, this article proposes an improved optimal gradient method, and redesigns the disturbance step size, which solves the oscillation problem well.

\subsection{Optimal Gradient Method}

The optimal gradient method is a relatively good variable step size control method, and its working principle is shown in Figure 4. Assuming that the system is currently working at point A, the disturbance voltage is $U(k)$, the slope of the tangent at point $A$ is $g_{k}$, and the relationship to $g_{k}$ is as follow:

$$
g_{k}=\left.\frac{\mathrm{d} P}{d U}\right|_{U=U(\mathrm{k})}
$$

Point $B$ is the next working point, and its corresponding disturbance voltage is $U(k+1)$. the magnitude of $U(k+1)$ is related to the disturbance voltage $U(k)$ and tangent slope $g_{k}$ of the current working point (point $A$ ) , Satisfy the following relationship:

$$
U(k+1)=U(k)+a_{k} g_{k}
$$

In formula (15), generally $a_{k}$ is a constant. It can be seen from formula (15) that the magnitude of the next disturbance voltage is completely determined by the state of the current operating point; at the same time, the closer to the maximum power point, the smaller the $g_{k}$ value and the smaller the disturbance step size; So for the optimal gradient method in the process of MPPT, the disturbance step size can be adjusted adaptively, thereby avoiding the oscillation problem and improving the tracking accuracy.

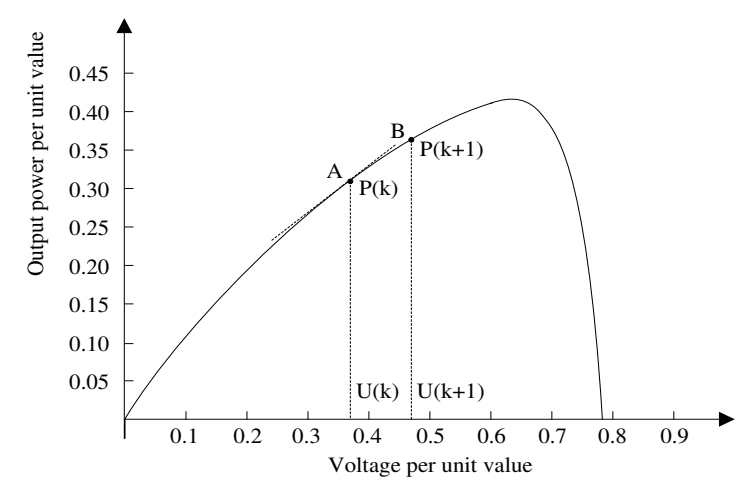

Figure 4 Optimal gradient method 


\subsection{Improved Optimal Gradient Method}

Although the optimal gradient method realizes the adaptive adjustment of the step size, this method also performs certain limitations. For example, in the early stage, when the disturbance is performed with a large step size, due to the large $g_{k}$ value, the calculated $U(k+1)$ value may exceed the open circuit voltage $U_{o c}$ of the solar panel; At the same time, the step size factor $a_{k}$ selected too large will cause the step size too large, which an oscillation phenomenon occurs. as we as the step size factor $a_{k}$ cannot adapt to the dynamic change of the $g_{k}$ value, and the dynamic performance is poor.

Since the photovoltaic cell is at the maximum power output, the corresponding output voltage is about $0.7 \mathrm{U}_{\mathrm{oc}}$, therefore, for defect 1 , the initial $U(\mathrm{k})$ value of the optimal gradient method can be set to $0.7 \mathrm{U}_{\mathrm{oc}}$, and then the conventional optimal gradient method Control, this control not only greatly reduces the $g_{k}$ value, but also reduces the tracking time.

For defect two, the step size factor $a_{k}$ can be redesigned. The design process is as follows.

The mathematical relationship between the step size factor $a_{k}$ and the working point tangent slope $g_{k}$ is constructed as an exponential function, as shown in formula (15).

$$
a_{k}=\beta\left(1-e^{-\left|g_{k}\right|}\right)
$$

In the formula, $\beta$ is a constant, $a_{k}$ is the step size factor, and $g_{k}$ is the slope of the tangent line at the operating point.

According to formula (15) and formula (16), the improved optimal gradient method perturbation voltage control equation can be obtained as:

$$
U(k+1)=U(k)+\beta\left(1-\mathrm{e}^{-\left|g_{k}\right|}\right) g_{k}
$$

According to formula (17), the step size is only related to the constant $\beta$ and the tangent slope of the operating point $g_{k}$ at this time.

The characteristic curve between the step size factor $a_{k}$ and the working point tangent slope $\mathrm{g}_{\mathrm{k}}$ is shown in Fig. 5.

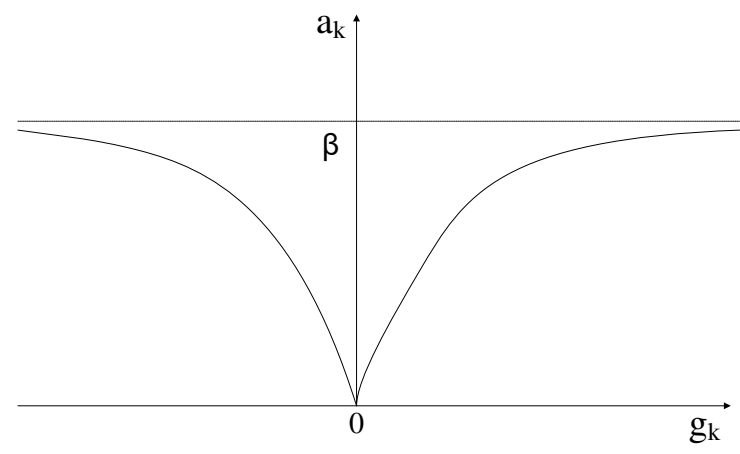

Figure 5 The characteristic curve between the step factor $a_{k}$ and the tangent slope $g_{k}$ of the operating point

It can be seen from Fig. 5 that the value of step size factor $a_{k}$ is limited between 0 and $\beta$, and as the absolute value of the tangent slope $g_{k}$ of the operating point decreases, the value of step size factor $a_{k}$ also decreases exponentially and tends to zero.

The improved optimal gradient method not only limits the step size and avoids the oscillation phenomenon, but also links the step size factor $a_{k}$ to the tangent slope $g_{k}$ of the operating point. The $a_{k}$ value can change dynamically with the change of the $g_{k}$ value, and the dynamic performance is improved. 


\section{Simulation analysis}

\subsection{System simulation model}

The system simulation model is shown in Figure 6. It is composed of PV subsystem, Boost circuit, load R, AlgoRithm1 subsystem, AlgoRithm2 subsystem, AlgoRithm3 subsystem and PWM subsystem. Among them, the AlgoRithm1 subsystem is a gradient method based on power prediction, the AlgoRithm2 subsystem is a gradient method based on improved power prediction, and the AlgoRithm3 subsystem is an improved optimal gradient method based on new power prediction. The gradient method step size factor of AlgoRithm1 subsystem and AlgoRithm2 subsystem are both a fixed value of 0.05 . The modified gradient method step factor size of AlgoRithm3 subsystem is variable, with an initial value of 0.05 . The PWM subsystem compares the reference voltage with the sawtooth wave to obtain the switching tube The simulation model parameters of PWM drive signal, PV subsystem and Boost circuit are shown in Table 1.

\begin{tabular}{ccccccr}
\multicolumn{7}{c}{ Tab.1 System simulation model parameters } \\
\hline PV & $\mathrm{I}_{\mathrm{m}}$ & $\mathrm{V}_{\mathrm{m}}$ & $\mathrm{V}_{\mathrm{oc}}$ & $\mathrm{I}_{\mathrm{se}}$ & $\mathrm{S}_{\mathrm{ref}}$ & $\mathrm{T}_{\text {ref }}$ \\
Battery & $4.96 \mathrm{~A}$ & $35.2 \mathrm{~V}$ & $44.2 \mathrm{~V}$ & $5.2 \mathrm{~A}$ & $1000 \mathrm{~W} / \mathrm{m}^{2}$ & $25^{\circ} \mathrm{C}$ \\
\hline Boost & $\mathrm{L}_{1}$ & $\mathrm{C}_{1}$ & $\mathrm{C}_{2}$ & $\mathrm{R}$ & & \\
Circuit & $5 \mathrm{e}^{-} 4 \mathrm{H}$ & $5 \mathrm{e}-4 \mathrm{~F}$ & $5 \mathrm{e}^{-} 4 \mathrm{~F}$ & $20 \Omega$ & & \\
\hline
\end{tabular}

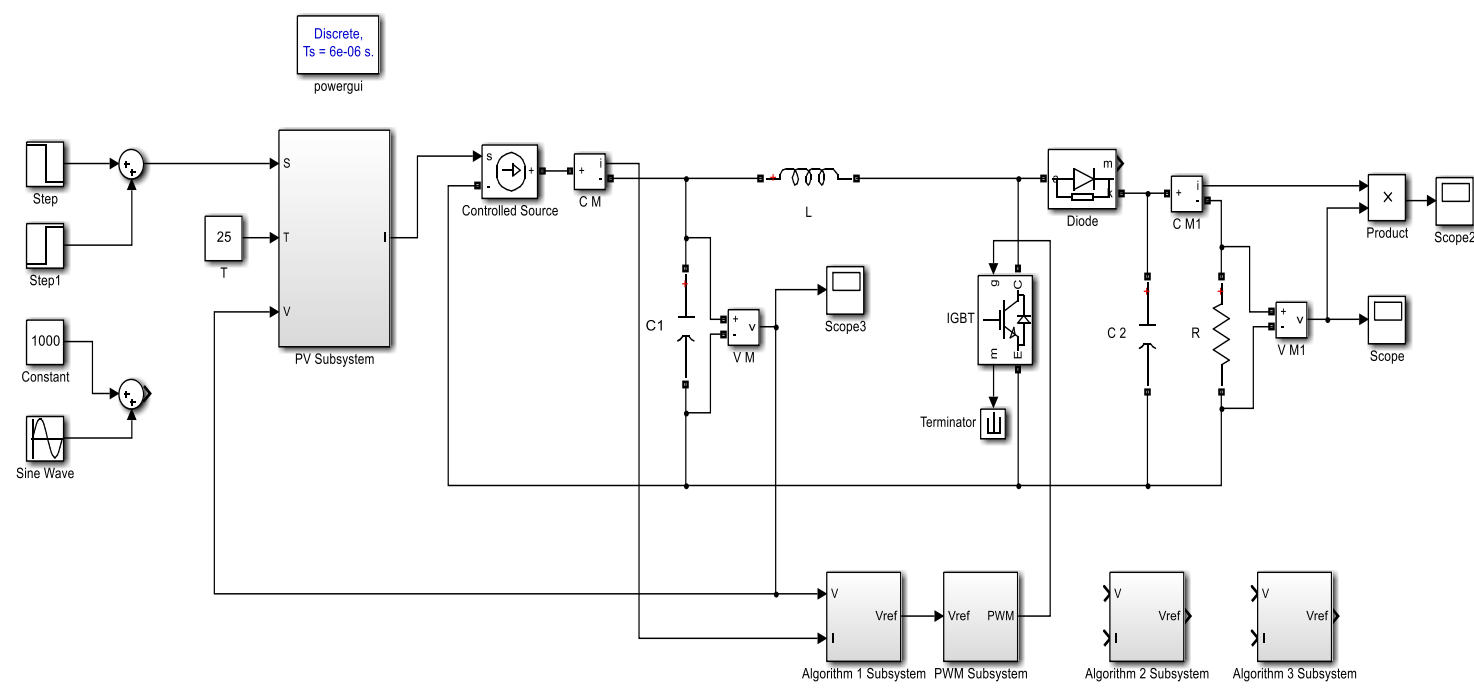

Figure 6 System simulation model

\subsection{Simulation analysis}

In order to simulate the MPPT tracking effect of the system under the conditions of rapid light changes, the temperature is constant at $25^{\circ} \mathrm{C}$ during the simulation, but the light conditions change rapidly. The two cases of light intensity step change and sinusoidal change were simulated respectively. The simulation time is $1 \mathrm{~s}$, the ode45 algorithm, and the sampling time is 6e-6s.

\subsubsection{Simulation when the light intensity changes step by step}

The given light intensity changes according to the step signal, as shown in Figure 7. $0 \sim 0.3 \mathrm{~s}$, the light intensity is constant at $1200 \mathrm{~W} / \mathrm{m} 2 ; 0.3 \sim 0.6 \mathrm{~s}$, the light intensity is constant at $800 \mathrm{~W} / \mathrm{m} 2$; $0.6 \sim 1 \mathrm{~s}$, the light intensity is constant at $1000 \mathrm{~W} / \mathrm{m} 2$. 


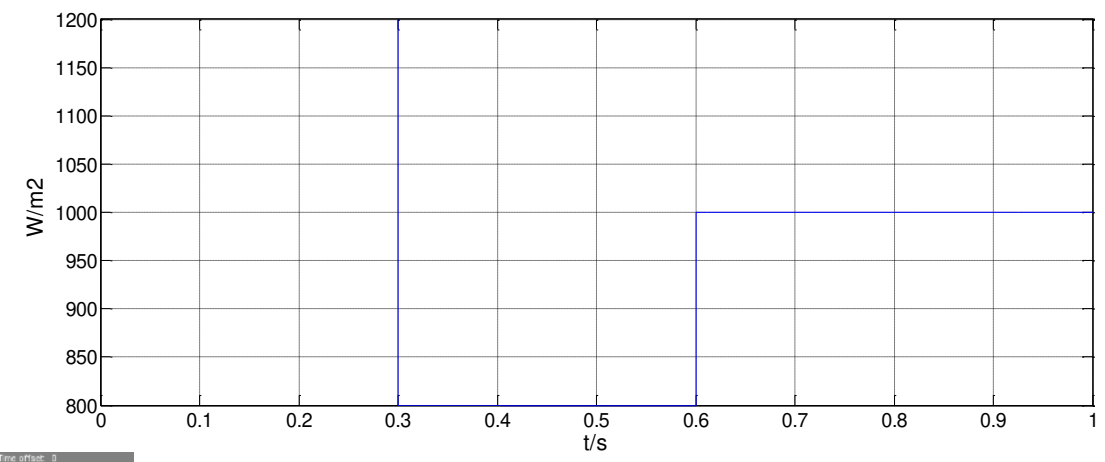

Figure 7 The given light intensity according to the step change

The MPPT simulation analysis was performed using the gradient method based on power prediction, the gradient method based on improved power prediction, and the improved optimal gradient method based on new power prediction. The simulation results are shown in Figure 8.

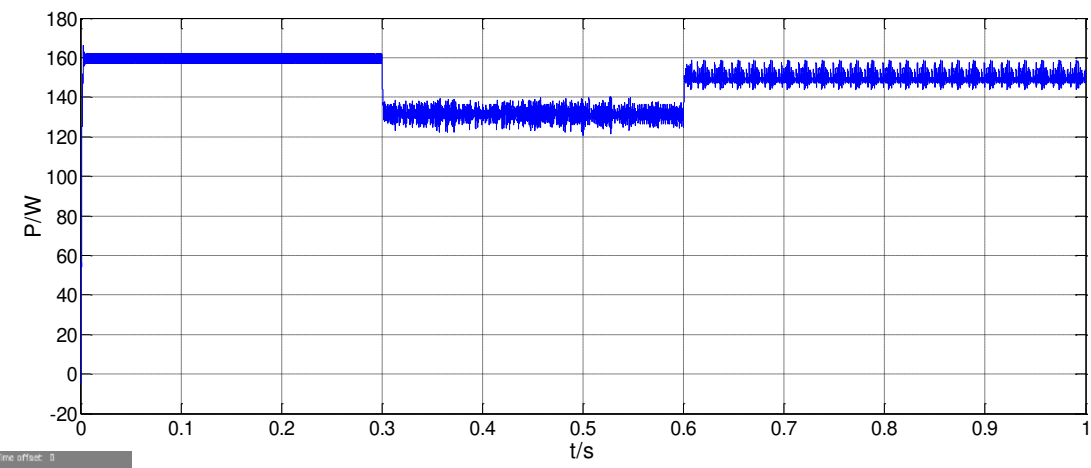

(a) Simulation results of gradient method based on power prediction

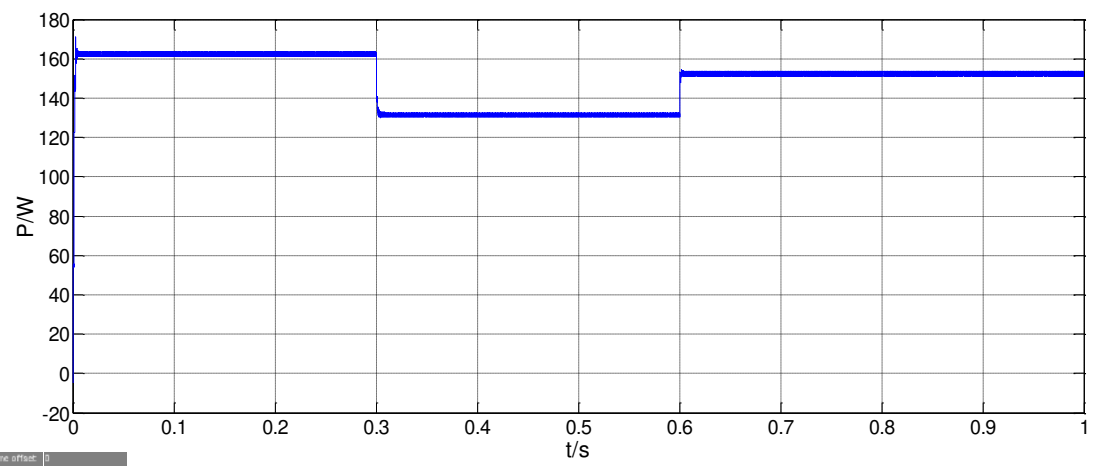

(b) Simulation results of gradient method based on improved power prediction

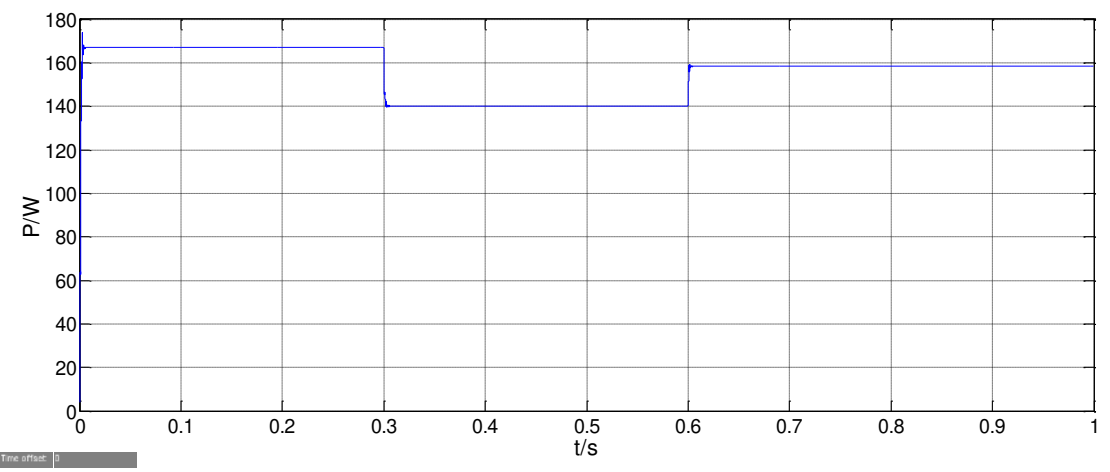

(c) Simulation results of the improved optimal gradient method based on the new power prediction

Figure 8 Simulation results of the three control algorithms $(a, b, c)$ when the light intensity changes step by step 
It can be seen from Figure $8(a): 0 \sim 0.3 s$, the average output power is $159.5 \mathrm{~W}, 0.3 \mathrm{~s} \sim 1 \mathrm{~s}$, there is obvious misjudgment phenomenon, $0 \sim 1 \mathrm{~s}$ there is obvious oscillation phenomenon. It can be seen from Figure8 (b): $0 \sim 0.3 \mathrm{~s}$, the average output power is $162.4 \mathrm{~W}, 0.3 \mathrm{~s} \sim 0.6 \mathrm{~s}$, the average output power is $131.5 \mathrm{~W}, 0.6 \mathrm{~s} \sim 1 \mathrm{~s}$, the average output power is $152.6 \mathrm{~W}, 0 \sim 1 \mathrm{~s}$ The misjudgment phenomenon has been eliminated, but there is still a small oscillation phenomenon. It can be seen from Figure 8 (c): $0 \sim 0.3 \mathrm{~s}$, the average output power is $166.9 \mathrm{~W}, 0.3 \mathrm{~s} \sim 0.6 \mathrm{~s}$, the average output power is $140.1 \mathrm{~W}, 0.6 \mathrm{~s} \sim 1 \mathrm{~s}$, the average output power is $158.3 \mathrm{~W}, 0 \sim 1 \mathrm{~s}$ Misjudgments and oscillations have been eliminated.

\subsubsection{Simulation when the light intensity changes sinusoidally}

The given light intensity changes according to the sinusoidal signal, as shown in Figure 9. The mathematical expression of the given light intensity is: $S=1000+200 \sin 4 \pi t$, the range of light intensity variation is $800 \sim 1200 \mathrm{~W} / \mathrm{m} 2$, and the period is $0.5 \mathrm{~s}$.

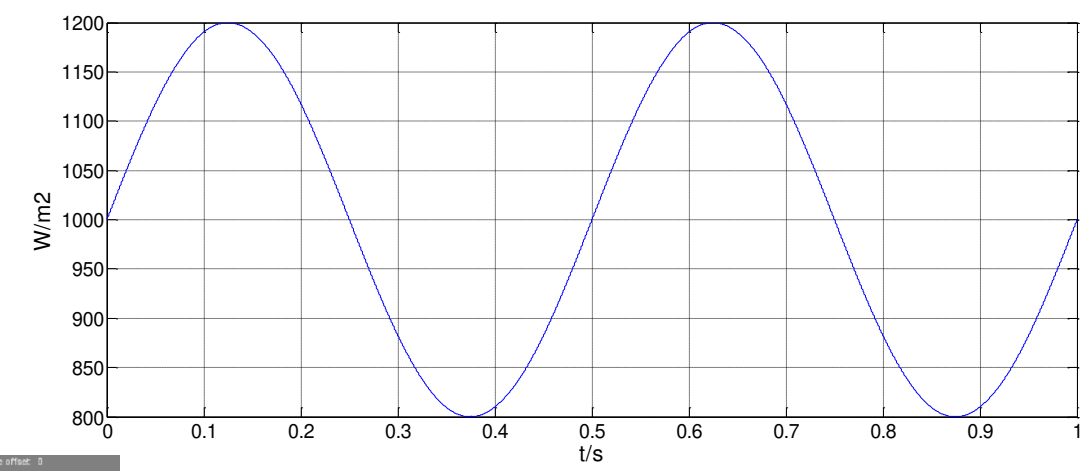

Figure 9 The light intensity of a given step change

The MPPT simulation analysis was performed using the gradient method based on power prediction, the gradient method based on improved power prediction, and the improved optimal gradient method based on new power prediction. The simulation results are shown in Figure 10.

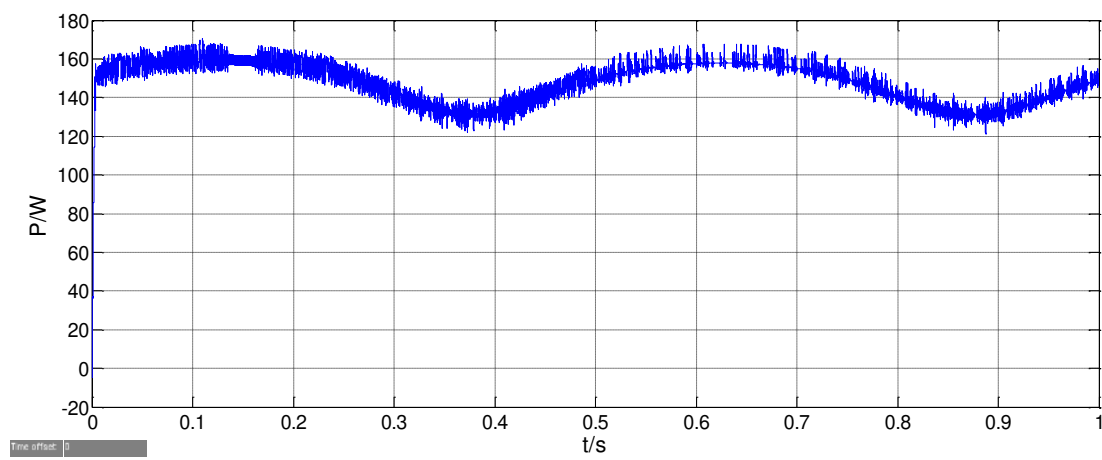

Figure a Simulation results of gradient method based on power prediction 


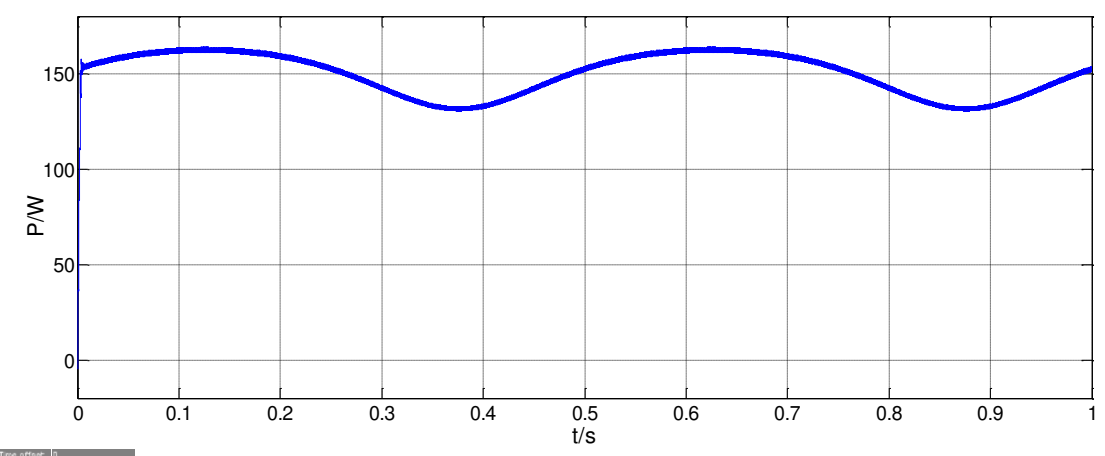

Figure $b$ Simulation results of gradient method based on improved power prediction

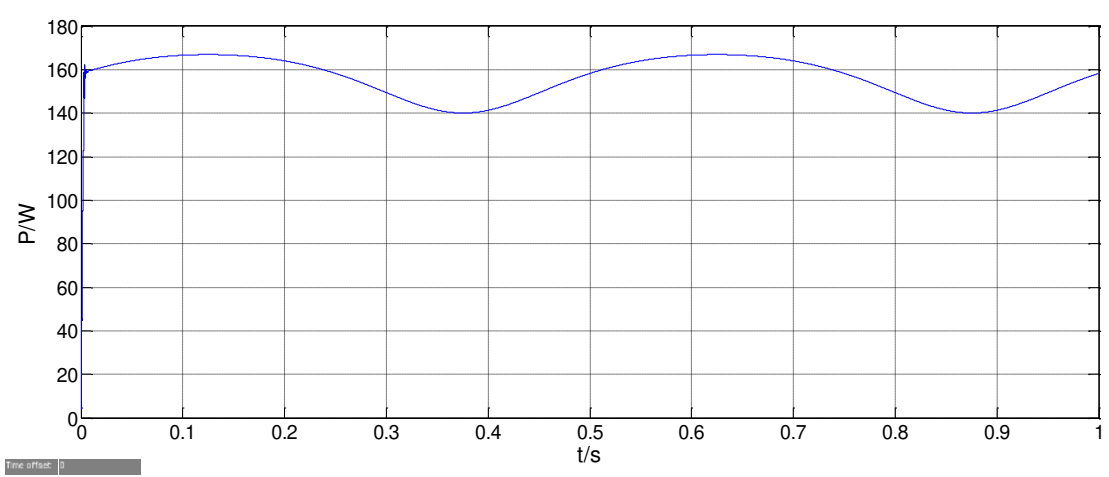

Figure $c$ Simulation results of the improved optimal gradient method based on the new power prediction

Figure 10 Simulation results of the three control algorithms $(a, b, c)$ when the light intensity changes sinusoidally

It can be seen from Figure10 (a) that there are obvious misjudgments and oscillations in the output power. It can be seen from Figure10 (b): the maximum output power is $163.8 \mathrm{~W}$, the minimum is $130.4 \mathrm{~W}$, the misjudgment phenomenon is eliminated, but there is still a small oscillation phenomenon. It can be seen from Figure10 (c) that the maximum output power is $166.9 \mathrm{~W}$, and the minimum is $140.1 \mathrm{~W}$. Misjudgment and oscillation are eliminated, and the output power is smooth and changes according to the sine law.

It can be seen from the simulation results of Fig. 8 and Fig. 10 that when the light intensity undergoes a step or sinusoidal change, the improved optimal gradient method using the new power prediction can not only eliminate the misjudgment phenomenon and oscillation phenomenon, but also the power calculation is more accurate, and the introduction of variable step size factor can make the system output more power, which can well realize the fast tracking of MPP.

\section{Summarize}

The MPP tracking problem of photovoltaic system under the condition of rapid change of light intensity is studied. The article first analyzes the principles and shortcomings of the traditional power prediction method and the improved power prediction method, and then proposes a novel power prediction method with a more accurate model, and then studies the optimal gradient method and proposes an improved optimal gradient method, finally the MPP tracking simulation analysis on the photovoltaic system is performed respectively using the gradient method based on power prediction, the gradient method based on improved power prediction, and the improved optimal gradient method based on new power prediction. The simulation results show that : When the light intensity changes rapidly, the improved optimal 
gradient method using the new power prediction can not only eliminate the misjudgment phenomenon and oscillation phenomenon, but also the power calculation is more accurate, and the introduction of variable step size factors can make the system output with greater power, fast tracking of MPP is well realized.

\section{References}

[1] Hua Zhihao, Zhu Wu, Guo Qiming. Overview of MPPT algorithm research on photovoltaic power generation system [J]. Power Technology, 2020, 44(12): 1855-1858

[2] Shen Shuqi, Li Guoyong. Research and design of maximum power point tracking of photovoltaic power generation system [J]. Computer simulation, 2021, 38(02): 72-75.

[3] Gao Lin. Research on Maximum Power Point Tracking Control Strategy of Photovoltaic Power Generation System [D]. Shandong: Shandong University of Science and Technology, 2018.

[4] He Lingqi. Research on the Maximum Power Point Tracking Algorithm of Photovoltaic Power Generation System [D]. Hunan: Hunan University, 2018.

[5] Xianwen Gao, Shaowu Li, Rongfen Gong. Maximum power point tracking control strategies with variable weather parameters for photovoltaic generation systems[J]. Solar Energy. 2013, 93: 357-367.

[6] Li Shaowu, Gao Xianwen. A VWP interval fuzzy MPPT control strategy for photovoltaic systems [J]. Acta Solar Energy, 2016, 37(05): 1167-1173.

[7] KANDEMIR E, CETIN N S, BOREKCI S. A comprehensive overview of maximum power extraction methods for PV systems [J] . Renewable and sustainable energy reviews, 2017, 78: 93-112

[8] KUMAR N, HUSSAIN I, SINGH B, et al. MPPT in dynamic condition of partially shaded PV system by using WODE technique [J] . IEEE transactions on sustainable energy, 2017, 8(3): 1204-1214.

[9] Peng Zhihui, Peng Yuchun, Zhou Hongming, et al. Optimal design of MPPT algorithm perturbation step size under rapid illumination changes [J]. Acta Solar Energy, 2020, 41 (08): 137-140.

[10] Liao Zhifan, Qi Xinmei, Zheng Shousen, et al. Analysis of photovoltaic system stability when light intensity changes rapidly[J]. Power System Technology, 2011, 35(07): 60-64.

[11] Mutoh N, Ohno M, Inoue T. A method for MPPT control while searching for parameters corresponding to weather conditions for PV generation systems[J]. IEEE Trans on Industrial Electronics, 2006, 53(04): 1055-1065.

[12] Gao Jilei, He Mingzhi, Zheng Qionglin. Simulation research on MPPT algorithm of photovoltaic grid-connected system under weather changes[J]. Journal of System Simulation. 2010, 22(04): 1000-1005

[13] Dezso Sera, Remus Teodorescu, Jochen Hantschel, Michael Knoll. Optimized Maximum Power Point Tracker for Fast-Changing Environmental Conditions [J]. IEEE Trans. Industrial Electronics(S0278-0046), 2008, 7, 55(07): 2629-2637.

[14] Belkaid A, Colak I, Isik O. Photovoltaic maximum power point tracking under fast varying of solar radiation[J]. Applied Energy, 2016, 179 (1): 523-530.

[15] Fan Ruixiang, Miao Jierong, Wang Wenbin, et al. Fast MPPT method for centralized solar inverter[J]. Journal of Electrical Machines and Control, 2019, 23 (07): 113-119.

[16] Xu Libin, Cheng Ruofa, Yang Jiajing, etc. Improved INC algorithm for rapid changes in light intensity[J]. New Technology of Electrical Engineering and Energy. 2020, 39(08): 56-64.

[17] Zhou Li, Xie Lei, Li Jie, et al. Research on Power Prediction Variable Step Perturbation Method in Photovoltaic MPPT [J]. Power Technology, 2020, 44(02): 230-234.

[18] Yuan Chenhu, Wang Kun, Liu Xiaoming, et al. Improved adaptive variable step size photovoltaic MPPT algorithm for power prediction [J]. Computer Simulation, 2021, 38(03): 34-38.

[19] Lin Yi, He Tongneng. Research on MPPT control strategy of variable step disturbance observation method based on power prediction [J]. Computer Measurement and Control, 2018, 26(05): 77-80. 
[20] Yang Tao, Lu Zhengyu. A new maximum power point tracking method in three-phase photovoltaic systems [J]. Journal of Zhejiang University, 2012, 46(08): 1490-1497.

[21] Zhang Junhong. Research on photovoltaic array and its key technologies [D]. Beijing: Beijing Jiaotong University, 2019.

[22] Yang Huan. Research and realization of the control algorithm for the maximum output power of photovoltaic modules [D]. Gansu: Lanzhou University, 2018. 